\title{
Suppression of multiple scattering effects in quasielastic light scattering by homodyne cross-correlation techniques
}

\author{
George D. J. Phillies \\ Department of Chemistry. The University of Michigan, Ann Arbor, Michigan 48109 \\ (Received 16 September 1980; accepted 25 September 1980) \\ By studying cross correlations in the intensity of light scattered by a fluid sample in different directions at \\ different times, it is possible to measure the dynamic scattering function $S(k, t)$ for the fluid. The multiple \\ scattering contribution to the time-dependent cross correlations is very substantially less than the multiple \\ scattering contribution to $S(\mathbf{k}, t)$ as obtained by a conventional single-detector quasielastic light scattering \\ system.
}

Quasielastic light scattering spectroscopy is commonly used in the study of bulk samples of weakly scattering fluids which are in thermal equilibrium. ${ }^{1}$ For such systems, in the absence of noise, ${ }^{2}$ the scattered light at a single point is characterized completely by its twotime intensity correlation function (spectrum) $S(\mathrm{k}, t)$. It is also possible to study cross correlations between light rays scattered by the sample in different directions. However, if homodyne detection is used, cross correlations between scattering intensities in different directions give at most the information that is already available from $S(\mathbf{k}, t) .^{3}$

In this note, it is shown that for the study of strongly scattering fluids one- and two-detector experiments are not equivalent, in that the double-scattering contributions to the spectrum and the cross spectrum are not the same. For the particular geometries considered here, multiple scattering has a far smaller effect on the twodetector cross-correlation spectrum than on the singledetector autocorrelation spectrum.

We first consider a conventional single-beam, singledetector experiment (Fig. 1). The incident light wave vector $\mathbf{k}_{I}$ illuminates the sample; some of it is scattered at point $A$ into light of wave vector $\mathbf{k}_{F}$, which goes to the detector. The scattering vector in the experiment is $\mathbf{k}=\mathrm{k}_{F}-\mathrm{k}_{I}$. The intensity of the singly scattered light $I_{1}(\mathrm{k}, t)$ is related to the positions $r_{i}(t)$ of the scattering particles (presumed identical) by

$$
I_{1}(k, t)=\left|E_{0}\right|^{2} \alpha^{2}\left|\sum_{i=1}^{N_{A}} \exp \left[i \mathbf{k} \cdot \mathbf{r}_{i}(t)\right]\right|^{2}
$$

$\alpha^{2}$ being the scattering cross section, $E_{0}$ the intensity of the incident beam, and $N_{A}$ the number of particles in the volume $A$.

Crosignani et al. ${ }^{1}$ demonstrate that multiple scattering contributions to the spectrum are dominated by uncorrelated events in which the scattering takes place in widely separated parts of the sample cell. In a typical event (Fig. 1), the light is scattered out of the incident beam at point $B$ and towards the detector at point $C$. The intermediate wave vector is $\mathbf{k}_{s}$, the scattering vector at the two events being $\mathbf{k}_{S}-\mathbf{k}_{I}=\mathbf{q}$ and $\mathbf{k}-\mathbf{q}$, respec-

\footnotetext{
a) Acknowledgment is made to The Donors of the Petroleum Research Fund, administered by the American Chemical Society, for their partial support of this work.
}

tively. The double scattering intensity corresponding to this pair of events is

$$
\begin{aligned}
I_{2}(\mathbf{k}, t)= & \left|E_{0}\right|^{2} \alpha^{4}\left|\sum_{i=1}^{N_{B}} \exp \left[i \mathbf{q} \cdot \mathbf{r}_{i}(t)\right]\right|^{2} \\
& \times\left|\sum_{j=1}^{N_{C}} \exp \left[i(\mathbf{k}-\mathbf{q}) \cdot \mathbf{r}_{j}(t)\right]\right|^{2},
\end{aligned}
$$

$N_{B}$ and $N_{C}$ referring to the number of particles in the scattering volumes at $B$ and $C$, respectively. The timedependent parts of the spectra corresponding to single scattering and to the specified double scattering event are

$$
\begin{aligned}
& \left\langle I_{1}(\mathbf{k}, t) I_{1}(\mathbf{k}, t+\tau)\right\rangle=\left|E_{0}\right|^{4} \alpha^{4} \\
& \quad \times\left|\left\langle\sum_{i, j=1}^{N} \exp \left\{i \mathbf{k} \cdot\left[\mathbf{r}_{i}(t)-\mathbf{r}_{j}(t+\tau)\right]\right\}\right\rangle\right|^{2}, \\
& \left\langle I_{2}(\mathbf{k}, t) I_{2}(\mathbf{k}, t+\tau)\right\rangle=\left|E_{0}\right|^{4} \alpha^{8} \\
& \quad \times \mid\left\langle\sum_{i, j=1}^{N_{B}} \exp \left\{i \mathbf{q} \cdot\left[\mathbf{r}_{i}(t)-\mathbf{r}_{j}(t+\tau)\right]\right\}\right\rangle^{2} \\
& \quad \times\left|\left\langle\sum_{m, n=1}^{N} \exp \left\{i(\mathbf{k}-\mathbf{q}) \cdot\left[\mathbf{r}_{m}(t)-\mathbf{r}_{n}(t+\tau)\right]\right\}\right\rangle\right|^{2} .
\end{aligned}
$$

The total double scattering spectrum is obtained by summing Eq. (3b) over all allowed pairs of volumes $(B, C)$, including the coincident volume $B \equiv C \equiv A$, using the appropriate value for $\mathbf{q}$ in each term of the sum.

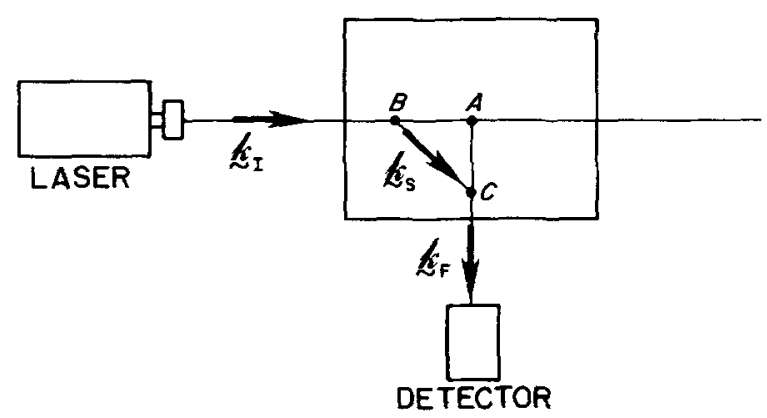

FIG. 1. Quasielastic light scattering apparatus, showing incident beam $\left(k_{I}\right)$, scattered light $\left(k_{F}\right)$ going to the detector, single scatt ering in volume $A$, and double scattering (with intermediate beam $k_{S}$ ) in volumes $B$ and $C$. 


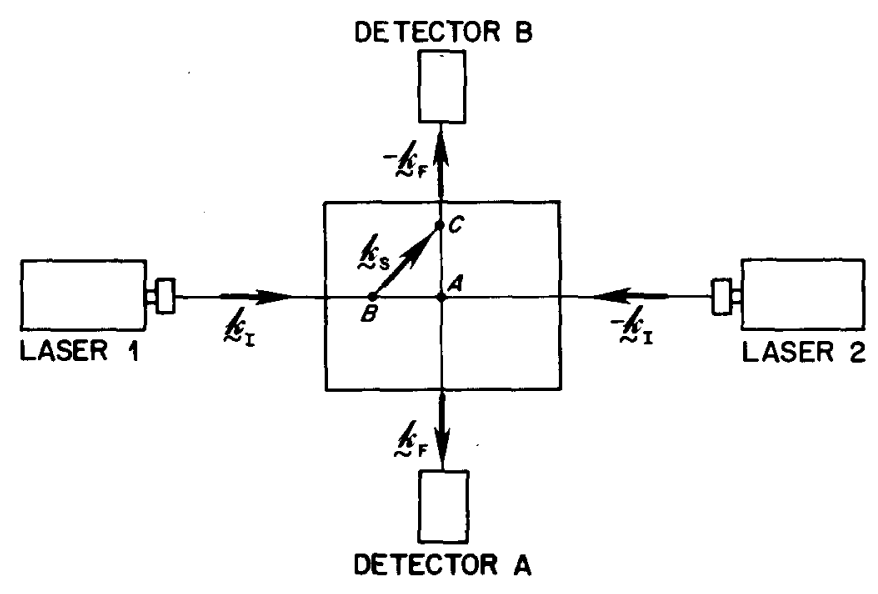

FIG. 2. The proposed two detector experiment. The geometry is planar. From symmetry the scattering wave vectors for laser 1 to detector $A$ and from laser 2 to detector $B$ are $\mathrm{k}$ and $-\mathrm{k}$, respectively; other labels are as in Fig. 1.

From Eq. (3b) one may justify the assertion that double scattering effects in bulk systems are dominated by noninteracting particles. In summing Eq. (3b) over possible volumes $B$ and $C$, one includes both interacting and noninteracting pairs. Denoting the numbers of particles in all allowed volumes $B$ and $C$ by $N_{B}$ and $N_{C}$, respectively, Eq. (3b) represents roughly $N_{B}^{4} N_{C}^{4}$ comparable terms. In a bulk system, each particle interacts with $\sim n$ of its neighbors $\left(n \ll N_{B}, N_{C}\right)$, so of all of the terms of Eq. (3b) only $\sim n^{2} N^{6}$ of them (drawn from the terms in which $B \equiv C$ ) represent cases in which, for example, particles $i$ and $m$ interact. Since the phases are random, the summations in Eqs. (3a) and (3b) grow roughly as the square root of the number of terms. Equation (3a) is therefore of order $\left(N E_{0}^{2} \alpha^{2}\right)^{2}$, while Eq. $(3 \mathrm{~b})$ is of order $\left(N E_{0}^{2} \alpha^{2}\right)^{2}\left(N \alpha^{2}\right)^{2}$. Double scattering events in which the first and second scatterings involve interacting particles represent fractionally $\sim n / N$ of the total number of double scattering events, and can normally be neglected by comparison with scattering by pairs of noninteracting particles.

We now consider a two-beam two-detector experiment
(Fig. 2). It would be possible to use one laser (plus a beam splitter and additional mirrors) instead of two lasers, at the possible price of seeing irrelevant interference effects between the beams, akin to those in a two-beam Michelson interferometer. Denoting the intensities at the two detectors by $I_{A}(t)$ and $I_{B}(t)$, the experiment measures $S_{x}(\mathrm{k}, \tau)=\left\langle I_{A}(t) I_{B}(t+\tau)\right\rangle$. Assuming that the two incident beams are well aligned and of equal intensity, and that the two detectors view the same volume of solution, the single-scattered intensities are

$$
\begin{aligned}
& I_{A 1}(t)=\left|E_{0}\right|^{2} \alpha^{2}\left|\sum_{i=1}^{N_{A}} \exp \left[i \mathbf{k} \cdot \mathbf{r}_{i}(t)\right]\right|^{2}, \\
& I_{B 1}(t)=\left|E_{0}\right|^{2} \alpha^{2}\left|\sum_{i=1}^{N_{A}} \exp \left[-i \mathbf{k} \cdot \mathbf{r}_{t}(t)\right]\right|^{2} .
\end{aligned}
$$

Since the magnitude of the complex exponential is taken, Eqs. (4a) and (4b) are identical. With this geometry, the single-scattering part of $S_{x}(\mathbf{k}, \tau)$ is

$$
\begin{aligned}
& \left\langle I_{A 1}(t) I_{B 1}(t+\tau)\right\rangle \\
& =\left\langle E_{0}^{4} \alpha^{4}\left|\sum_{i, j=1}^{N_{A}} \exp \left\{i \mathrm{k} \cdot\left[\mathbf{r}_{i}(t)-\mathbf{r}_{j}(t+\tau)\right]\right\}\right|^{2}\right\rangle,
\end{aligned}
$$

i.e., the new geometry measures the same dynamic structure factor $S(k, t)$ that the single-detector experiment does. There is a slight difference. It is readily shown that $\left\langle\exp \left[i \mathbf{k} \cdot \mathbf{r}_{i}(t)+i \mathbf{k}^{\prime} \cdot \mathbf{r}_{j}(\tau)\right]\right\rangle=0$ for $\mathbf{k}^{\prime} \neq-\mathbf{k}$. In measuring $S(\mathbf{k}, \tau)$, the same scattering vector is used at $t$ and $t+\tau$, the $-\mathbf{k}$ being obtained when the intensity of the scattered field is found. In obtaining $S_{x}(\mathbf{k}, t)$, one beam-detector pair obtains $\mathbf{k}$, and a separate beamdetector pair obtains $\mathbf{k}^{\prime}=-\mathbf{k}$.

One may also consider how double scattering affects $S_{\mathbf{x}}(\mathbf{k}, \tau)$. In a representative pair of events, one photon passes from laser 1 through points $B$ and $C$ to detector $A$, while a second photon passes from laser 2 through points $B$ and $C$ to detector $B$. For the first photon, the scattering vectors are $\mathbf{k}_{S}-\mathbf{k}_{I}=\mathbf{q}$ at $B$ and $\mathbf{k}_{F}-\mathbf{k}_{S}=\mathbf{k}-\mathbf{q}$ at $C$. For the second photon, the scattering vectors are $\mathbf{k}_{S}-\left(-\mathbf{k}_{I}\right)=\mathbf{q}+2 \mathbf{k}_{I}$ at $B$ and $-\mathbf{k}_{F}-\mathbf{k}_{S}=\mathbf{k}-\mathbf{q}-2 \mathbf{k}_{F}$ at $C$. The contribution of double scattering to $S_{x}(k, \tau)$ is

$$
\begin{aligned}
\left\langle I_{A 2}(t) I_{B 2}(t+\tau)\right\rangle=\left\langle E_{0}^{4} \alpha^{8}\right. & \left|\sum_{i=1}^{N_{B}} \exp \left[i \mathbf{q} \cdot \mathbf{r}_{i}(t)\right]\right|^{2}\left|\sum_{j=1}^{N_{B}} \exp \left[i\left(\mathbf{q}+2 \mathbf{k}_{I}\right) \cdot \mathbf{r}_{j}(t+\tau)\right]\right|^{2} \\
& \left.\times\left|\sum_{m=1}^{N_{C}} \exp \left[i(\mathbf{k}-\mathbf{q}) \cdot \mathbf{r}_{m}(t)\right]\right|^{2}\left|\sum_{n=1}^{N_{C}} \exp \left[+i\left(\mathbf{k}-\mathbf{q}-2 \mathbf{k}_{F}\right) \cdot \mathbf{r}_{n}(t+\tau)\right]\right|^{2}\right\rangle .
\end{aligned}
$$

In Eq. (6), $i$ and $j$ are in volume $B$, and $m$ and $n$ in volume $C$. Even if volumes $B$ and $C$ overlap, $(i, j)$ and $(m, n)$ (which may be pairwise identical) are separated by a vector parallel to $\mathbf{k}_{s}$. In Eq. (6), it is important that the indices $i, j, m$, and $n$ do not all refer to the same particle $(i=j=m=n$ is a single scattering event). Equation (6) then contains only terms like

$$
\begin{aligned}
& \left|E_{0}\right|^{4} \alpha^{8} \mid\left\langle\sum_{t, j=1}^{N_{B}} \exp \left\{i \mathbf{q} \cdot\left[\mathbf{r}_{i}(t)-\mathbf{r}_{f}(t+\tau)\right]-2 i \mathbf{k}_{I} \cdot \mathbf{r}_{j}(t+\tau)\right\}\right. \\
& \left.\times \sum_{m, n=1}^{N_{C}} \exp \left\{i(\mathbf{k}-\mathbf{q}) \cdot\left[\mathbf{r}_{m}(t)-\mathbf{r}_{n}(t+\tau)\right]+2 i \mathbf{k}_{F} \cdot \mathbf{r}_{n}(t+\tau)\right\}\right\rangle\left.\right|^{2}
\end{aligned}
$$


As long as the volumes $B$ and $C$ are distinct, the two exponentials may be separately averaged. $\left\langle\exp \left[2 i \mathbf{k}_{\boldsymbol{I}}\right.\right.$ - $\left.\left.\mathbf{r}_{j}(t+\tau)\right]\right\rangle$ and $\left\langle\exp \left[-2 i \mathbf{k}_{F} \cdot \mathbf{r}_{n}(t+\tau)\right]\right\rangle$ vanish by trans lational invariance if volumes $B$ and $C$ are large. (For diffraction-limited diameters of the scattering volume, as is rarely encountered in practice, this argument would need modification.)

If the volumes $B$ and $C$ overlap, double scattering can contribute to the cross spectrum, namely, for overlapping volumes a representative term of Eq. (6) can be written

$$
\begin{aligned}
& \left|E_{0}\right|^{4} \alpha^{8} \mid \sum_{i, j, m, n=1}^{N}\left\langle\exp \left\{2 i \mathbf{k}_{F} \cdot\left[\mathbf{r}_{j}(t)-\mathbf{r}_{n}(t+\tau)\right]\right\}\right. \\
& \times \exp \left\{i \mathbf{k} \cdot\left[\mathbf{r}_{m}(t+\tau)+\mathbf{r}_{n}(t+\tau)-2 \mathbf{r}_{j}(t)\right]\right\} \\
& \left.\times \exp \left\{i \mathbf{q} \cdot\left[\mathbf{r}_{i}(t)+\mathbf{r}_{j}(t)-\mathbf{r}_{m}(t+\tau)-\mathbf{r}_{n}(t+\tau)\right]\right\}\right)\left.\right|^{2} .
\end{aligned}
$$

For the average to be nonzero, the first exponential requires $j$ and $n$ to interact; the second exponential then requires $j, n$, and $m$ be close enough to interact. The final exponential consequently requires $j, m, n$, and $i$ to interact. This is only possible if $i, j, m$, and $n$ are in the same microscopic volume. If volumes $B$ and $C$ overlap, $i=j \neq m=n$, corresponding to an interacting pair of particles, is also allowed.

If volumes $B$ and $C$ overlap, it is also possible to have a pair of double scattering events in which one ray passes from laser 1 to $B$ to $C$ to detector $A$ and the other ray passes from laser 2 to $C$ to $B$ to detector $B$. The scattering vectors for this series are $\mathbf{q}, \mathbf{k}-\mathbf{q},-\mathbf{q}$, and $-(\mathbf{k}-\mathbf{q})$, respectively; a representative double scattering term of this sort is

$$
\begin{aligned}
& \left|E_{0}\right|^{2} \alpha^{4} \mid \sum_{i, j, m, n=1}^{N} \exp \left\{i \mathbf { q } \cdot \left[\mathbf{r}_{i}(t)+\mathbf{r}_{n}(t+\tau)\right.\right. \\
& \left.\left.\quad-\mathbf{r}_{j}(t+\tau)-\mathbf{r}_{m}(t)\right]\right\}\left.\exp \left\{i \mathbf{k} \cdot\left[\mathbf{r}_{j}(t)-\mathbf{r}_{n}(t+\tau)\right]\right\}\right|^{2} .
\end{aligned}
$$

Here particles $i$ and $n$, and particles $j$ and $m$, may be close to each other (or the same) but the pairs $(i, n)$ and $(j, m)$ must be separated by a vector parallel to $\mathbf{k}_{s}$. Term $(9)$ is only nonvanishing if $(i, n)$ and $(j, m)$ are within the same correlation volume.

By comparison with the discussion following Eqs. (3) the single-scattering contribution to $S_{x}(\mathbf{k}, \tau)$ is of order $\left(N E_{0}^{2} \alpha^{2}\right)^{2}$, which is the same as the single scattering contribution to $S(\mathbf{k}, \tau)$. In contrast, the double scattering contribution to $S_{x}(\mathbf{k}, t)$ is of order $\left(N E_{0}^{2} \alpha^{2}\right)^{2}\left(n \alpha^{2}\right)^{2}, n$ being the number of particles interacting with a given particle. The double-scattering contribution to $S_{x}(\mathbf{k}, \tau)$ is therefore less than its contribution to $S(\mathbf{k}, \tau)$ by a factor of $(n / N)^{2}$. This reduction in double-scattering effects by use of a two-detector system should permit study of more strongly scattering systems than is elsewise possible. Several experimental considerations for a two-detector experiment may be noted:

(a) For the experiment to work, the scattering vectors $\mathbf{k}$ and $-\mathbf{k}$ defined by the two beam-detector combinations must be the same, i.e., they must be matched to within $\lambda / 10$ over the scattering volume. For visible light, $\lambda \sim 5000 \AA$; a scattering volume of dimensions $\mathbf{r}_{0}$ $\sim 100 \mu \mathrm{m}$ is not unreasonable. These values require beam alignment to within $\Delta \theta \sim \lambda / 10 \mathbf{r}_{0} \equiv \dot{0} .5 \mathrm{mrad}$, which is difficult, but not impossible.

(b) The analysis has implicitly assumed that the incident and scattered laser beams are plane waves, requiring spatial filtering of the incident laser beams. The planarity requirement also constrains the focal lengths of focusing and collecting lenses, since too short a focal length will lead to incident or scattered waves which are manifestly spherical while inside the scattering volume.

(c) In the detector geometry of Fig. 2, there is nothing to prevent photons from being scattered into the "wrong" detector, e.g., photons of laser 1 can be scattered into detector $B$. This introduces a second scattering vector $k^{\prime}=\mathbf{k}_{I}+\mathbf{k}_{F}$. For $90^{\circ}$ scattering, the effect is irrelevant, since $|\mathbf{k}|=\left|\mathbf{k}^{\prime}\right|$; at other scattering angles, $S_{x}(\mathbf{k}, \tau)$ is the sum of $S(\mathbf{k}, \tau)$ and $S\left(\mathbf{k}_{x}^{\prime}, \tau\right)$, making data analysis more difficult. If one uses two lasers of different wavelengths, by placing appropriate interference filters in front of the detectors one may insure that each detector will only be sensitive to light scattered through a unique scattering vector.

A two-detector scheme similar to Fig. 2 was used by Griffith and Pusey ${ }^{4}$ to study rotational diffusion of single tobacco mosaic virus particles. In this experiment, only a single incident beam was used, the spectrum being due to the fluctuations in the total number and orientation of particles in the beam. By contrast, in the experiment proposed here, fluctuations in the number of particles in the beam are taken to be unimportant, $S_{x}(\mathbf{k}, \tau)$ arising from changes in the relative positions of a fixed number of scatterers. In the language of Griffith and Pusey, we have proposed a "Gaussian" two-detector correlation experiment, while their experiment relied on the nonGaussian nature of light scattered by an exceedingly small number of particles.

${ }^{1}$ B. Crosignani, P. DiPorto, and M. Bertolotti, Statistical Properties of Scattered Light (Academic, New York, 1975).

${ }^{2}$ In the presence of noise, higher correlation functions may be experimentally useful. G. D. J. Phillies, J. Chem. Phys. $72,6123(1980)$.

${ }^{3}$ Compare Ref. 1. With heterodyne detection, multidetector experiments may give results beyond $S(\mathrm{k}, t), \mathrm{G}, \mathrm{D}$. J. Phillies, Mol. Phys. 32, 1695 (1976).

${ }^{4}$ W. G. Griffith and P. N. Pusey, Phys. Rev. Lett. 43, 1100 (1979). 